\title{
MACÁBOT: Prototipo de Vehículo Autónomo de Superficie (ASV)
}

\section{MACÁBOT: Prototype Autonomous Surface Vehicle (ASV)}

\section{Gerardo G. Acosta}

Centro de Investigaciones en Física e Ingeniería del Centro -Universidad Nacional del Centro de la Provincia de Buenos Aires, Consejo Nacional de Investigaciones Científicas y Técnicas - Argentina gacosta@fio.unicen.edu.ar

\section{Bruno V. Menna}

Centro de Investigaciones en Física e Ingeniería del Centro -Universidad Nacional del Centro de la Provincia de Buenos Aires, Consejo Nacional de Investigaciones Científicas y Técnicas - Argentina bmenna@fio.unicen.edu.ar

\section{Roberto J. de la Vega}

Universidad Nacional del Centro de la Provincia de Buenos Aires- Argentina. rdelavega@fio.unicen.edu.ar

\section{Luis M. Arrien}

Universidad Nacional del Centro de la Provincia de Buenos Aires- Argentina. larrien@fio.unicen.edu.ar

\section{Hugo J. Curti}

Universidad Nacional del Centro de la Provincia de Buenos Aires- Argentina. hcurti@fio.unicen.edu.ar

\section{Sebastián A. Villar}

Centro de Investigaciones en Física e Ingeniería del Centro -Universidad Nacional del Centro de la Provincia de Buenos Aires, Consejo Nacional de Investigaciones Científicas y Técnicas - Argentina svillar@fio.unicen.edu.ar 


\title{
Roberto C. Leegstra
}

Universidad Nacional del Centro de la Provincia de Buenos Aires- Argentina.

rleegstra@fio.unicen.edu.ar

\section{Mariano De Paula}

Centro de Investigaciones en Física e Ingeniería del Centro -Universidad Nacional del Centro de la Provincia de Buenos Aires, Consejo Nacional de Investigaciones Científicas y Técnicas - Argentina mdepaula@fio.unicen.edu.ar

\section{Ignacio Carlucho}

Centro de Investigaciones en Física e Ingeniería del Centro -Universidad Nacional del Centro de la Provincia de Buenos Aires, Consejo Nacional de Investigaciones Científicas y Técnicas - Argentina icarlucho@fio.unicen.edu.ar

\section{Franco J. Solari}

Centro de Investigaciones en Física e Ingeniería del Centro -Universidad Nacional del Centro de la Provincia de Buenos Aires, Consejo Nacional de Investigaciones Científicas y Técnicas - Argentina fsolari@fio.unicen.edu.ar

\section{Alejandro F. Rozenfeld}

Centro de Investigaciones en Física e Ingeniería del Centro -Universidad Nacional del Centro de la Provincia de Buenos Aires, Consejo Nacional de Investigaciones Científicas y Técnicas - Argentina arozenfeld@fio.unicen.edu.ar

\section{Resumen}

En este artículo se presentan las decisiones de diseño y construcción de un robot móvil autónomo que navega en la superficie acuática, conocido como vehículo autónomo de superficie (ASV por sus siglas en inglés). Fue casi totalmente desarrollado y armado en los laboratorios del Núcleo INTELYMEC-CIFICEN, de la Universidad Nacional del Centro de la Prov. de Buenos Aires, Argentina en cooperación con una empresa interesada en su comercialización. Este prototipo, llamado MACÁBOT ha sido exitosamente probado en aguas calmas. Desde el punto de vista de I+D, se lo empleará como una plataforma experimental para nuevos algoritmos y tecnología subacuática. Su construcción mecánica, la electrónica de bajo nivel, como también su arquitectura de software es explicada. También se presentan las primeras pruebas acuáticas con una discusión sobre las mismas.

Palabras Claves: ASV, Autónomo, Vehículo.

\begin{abstract}
In this article the design and construction decisions of a mobile autonomous robot that navigates in water surface, known as autonomous underwater vehicle (ASV), are presented. It was almost totally developed and assembled in the laboratories of INTELYMEC-CIFICEN, Universidad Nacional del Centro de la Prov. de Buenos Aires, Argentina in cooperation with a national enterprise interested in its commercialization. This prototype called MACÁBOT has been successfully tested in calm waters. From the standpoint of $\mathrm{R}+\mathrm{D}$ it will be employed
\end{abstract}


as an experimental platform for new algorithms and underwater technology. Its mechanical construction, the low level electronic designs as well as its software architecture is explained. Wet trials are also presented with further discussion about them.

Keywords: ASV, Autonomous, Vehicle

\section{INTRODUCCIÓN}

Los robots autónomos que se desplazan en la superficie del agua han estado teniendo un discreto pero persistente rol en los ambientes marinos, comenzando a ser más protagonistas en los últimos años. Se los refiere comúnmente como vehículos autónomos de superficie o ASV por sus siglas en inglés. Últimamente, se está prestando más atención a los ASV para diferentes y variados tipos de servicios tales como inspecciones de infraestructura sumergida, muestreo oceanográfico, batimetrías, estudios de ecosistemas acuáticos, seguridad portuaria, operaciones de búsqueda y rescate, entre otras [1-2]. En gran medida también como partes del sistema de posicionamiento de vehículos subacuáticos, transfor-mándose en boyas virtuales de estos sistemas para multi-lateralización de eco [3].

Estos ASV disponen de la interesante característica de combinar la habilidad de transportar una gran variedad de sensores bien interconectados con un gran ancho de banda para la comunicación a estaciones costeras o embarca-ciones, lo cual es mucho más difícil en los robots autónomos subacuáticos. Esta característica los convierte en un sistema de avanzada para la telepresencia en el mar. Mientras navegan en la superficie pueden transportar sensores tales como sonares de barrido lateral, ecosondas, y sonares de largo alcance, permitiéndoles también escrutar las profundidades.

Para la construcción de un robot autónomo de este tipo, capaz de completar una misión en un ambiente real, previamente desconocido y frecuentemente hostil, algunos bloques constructivos deben estar necesariamente presentes [4]. En efecto, es necesario proveerlo de un conjunto de sensores a bordo que determinen la percepción de su entorno o sistema de percepción (PS) y su propia presencia en ese entorno, codificada en posición y actitud, denominado sistema de navegación (NS). Asimismo se requieren un sistema de guiado (GS) para desplegar una trayectoria a partir de requerimientos de módulos externos, como una interfaz de usuario o un módulo de alto nivel que modifique la misión del robot, y lazos de control que le permitan describir esta trayectoria y tareas propuestas con un mínimo error. Estos últimos forman parte del módulo de sistema de control (CS) [5-6]. Finalmente, para dotar al robot de autonomía, esto es, poder realizar sus propias decisiones de acuerdo a la situación de su entorno y sin intervención humana, se requiere un módulo de mayor nivel jerárquico en la pirámide de control, un estrato de control supervisorio [7]. Este módulo será el responsable de interpretar la misión programada por el usuario y adaptarla en función la evasión de obstáculos, cambio de prioridades contradictorias, replanificación de objetivos frente a incidencias y fallas, entre otras. Este módulo denominado sistema de planificación dinámica (DMPS) puede de este modo generar puntos de paso con velocidad y actitud programada para el robot, en una forma adaptable, de modo tal que el robot exhiba un comportamiento inteligente. A partir de estos puntos de paso, el GS calcula las referencias para los lazos del CS. Como puede apreciarse a partir de esta breve descripción, una vez diseñado el flujo de datos entre módulos, cada uno podrá abordarse con diferentes y heterogéneas técnicas y algoritmos resultantes, convirtiendo a todo el robot en una plataforma experimental 
muy rica para el descubrimiento de nuevos conocimientos.

Este artículo presenta estas ideas plasmadas en el desarrollo de un prototipo de ASV que denominamos MACÁBOT, a semejanza del Macá, un ave acuática sudamericana. La siguiente sección provee una descripción mecánica y constructiva, con los sensores y propulsores seleccionados durante la etapa de diseño. La siguiente sección III presenta la electrónica y el módulo de comunicaciones del prototipo, mientras que la sección IV se enfoca principalmente la arquitectura de software para operar en un ambiente no estructurado. El trabajo se completa con algunos experimentos iniciales en aguas tranquilas y unas conclusiones preliminares.

\section{DESCRIPCIÓN DE LA PLATAFORMA}

El ASV Macábot está constituido por un chasis de acero inoxidable que sostiene dos propulsores frecuentemente usados en buceo, marca TORPEDO 2000TM, manejados con placas MD03 con un puente $\mathrm{H}$, capaces de proveer una corriente de hasta $20 \mathrm{~A}$ a cada motor operando a $12 \mathrm{~V}$, lo que permite obtener una potencia acorde a las aplicaciones del mundo real y controlables con un micro-controlador PIC-16F872 que acepta comunicación I2C. Sus dimensiones físicas son: Eslora: 1,70m; Manga:1,10m; Puntal: 0,90m. Los propulsores están constituidos por motores de c.c. sumergibles hasta $52 \mathrm{~m}$. de profundidad y capaces cada uno de alcanzar hasta 1,7 nudos arrastrando una masa de hasta 10kg. El chasis integra además dos flotadores de triple capa de PVC hecho con adhesivos y soldadura de alta frecuencia, como se aprecia en la Fig. 1. Con ambos motores es posible determinar el rumbo sin la necesidad de un timón. En este mismo chasis se montó una caja estanca de plástico (450x300x200mm) con la electrónica, y dos cajas estancas más del mismo tipo pero de menores dimensiones $(300 \times 300 \times 200 \mathrm{~mm})$, para alojar las baterías, una para la electrónica y otra para los motores. Las baterías son blindadas de plomo-ácido libre de mantenimiento, de 12V 7Ah, conectadas en paralelo, dos en cada caja totalizando cuatro a bordo.

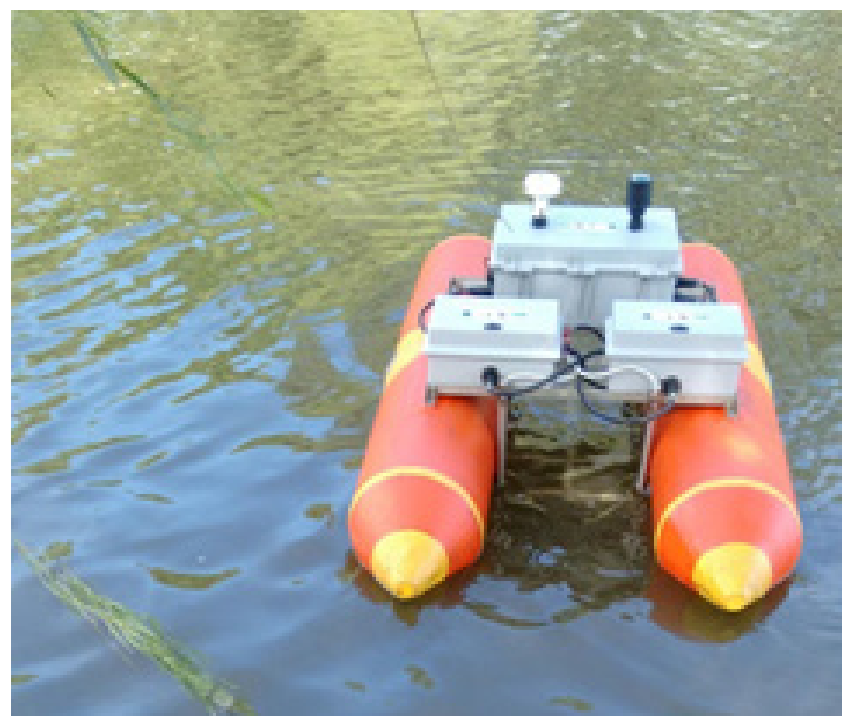

Fig. 1. ASV Macábot, con chasis de acero inoxidable y cajas estancas para electrónica y baterías. 
Debajo de la superficie, dispone de una quilla de aluminio, aislada del chasis para evitar que funcione como ánodo de sacrificio, donde se montan los sensores para la misión, como sonar de barrido lateral o ecosonda mono-haz mecánicamente escaneada, o cualquier otro sensor útil para la misión (payload), como se aprecia en la Figura 2. Esto lo convierte en una plataforma multipropósito adaptable a diferentes aplicaciones.
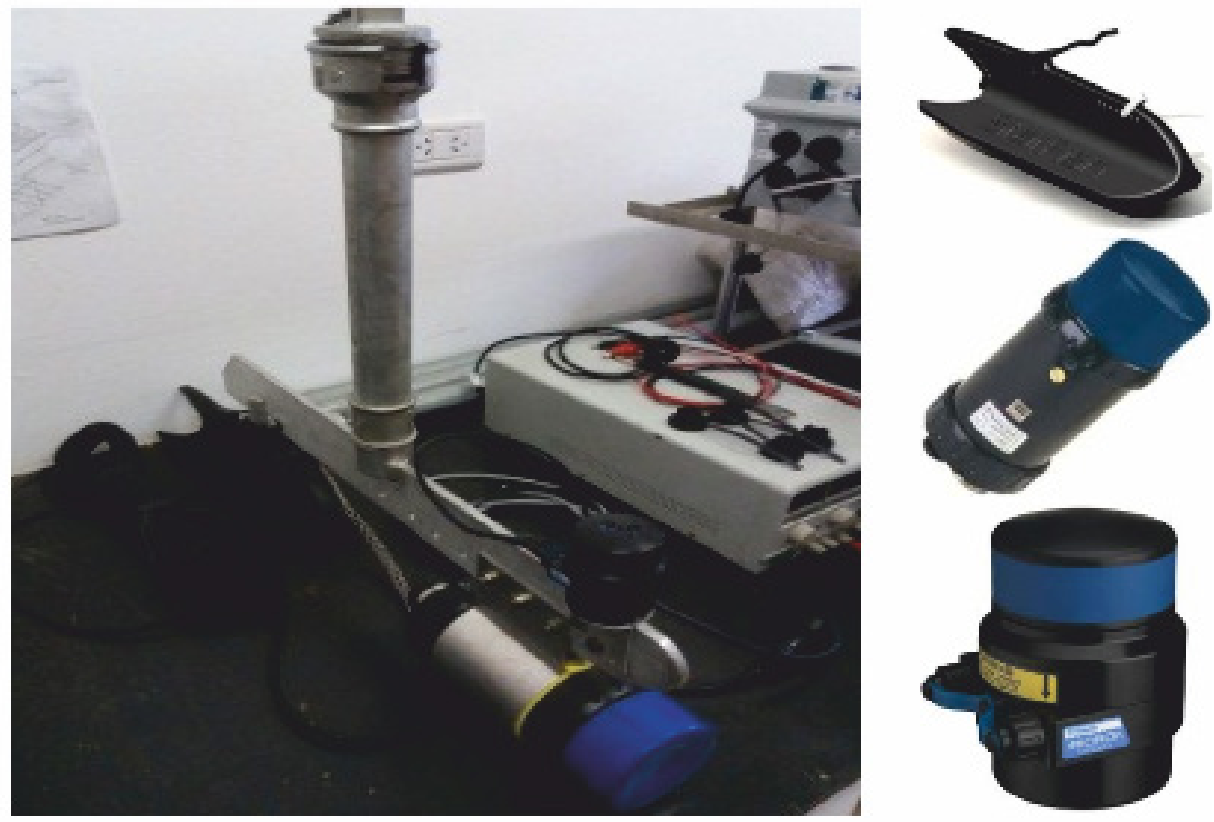

Fig. 2. sensores acústicos a bordo de la quilla (izquierda) consistentes en: (derecha desde arriba hacia abajo) sonar de barrido lateral StarFish, ecosonda SuperSeaKing y ecosonda Micron, de la firma TritechTM

\section{MÓDULOS DE HARDWARE}

A. Sistemas de procesamiento de alto y bajo nivel

La electrónica del ASV se diseñó en dos sistemas de procesamiento principales: a) de alto nivel y b) de bajo nivel. El sistema de alto nivel lo constituye una CPU Advantech ${ }^{\circledR}$ ARK1380, con procesador Intel Celeron $1 \mathrm{GHz}-4 \mathrm{USB}-2 \mathrm{COM}-1 \mathrm{LAN}$, fanless, de un consumo aproximado de $35 \mathrm{w}$, y SSD de $64 \mathrm{~Gb}$. A esta CPU se conectan directamente por puertos serie universales un GPS y una unidad de navegación inercial, para constituir junto con la brújula, los proveedores de datos al sistema de navegación (NS). También se conectan a través de sus puertos serie los dispositivos necesarios para la percepción del robot (PS), como por ejemplo los sonares mencionados en la sección anterior (Fig. 2).

El sistema de bajo nivel dispone de un microcontrolador MSP430F247 de Texas Instruments@ como procesador central y encargado de las comunicaciones entre los nodos sensores y actuadores. Se trata de una configuración maestro-esclavos sobre un bus LIN (Local Interconnect Network), subconjunto del protocolo CAN (Controller Area Network). Los nodos esclavos desarrollados y operativos hasta la fecha son brújula, manejo de energía, 
placas controladoras de motores y emergencia. Ambos subsistemas de alto y bajo nivel se comunican a través del puerto serie USB.

\section{ARQUITECTURA DE SOFTWARE}

La programación del robot refleja los lineamientos establecidos para la robótica móvil autónoma, que fueron presentados en la sección introductoria. Su implementación fue realizada sobre el "Robot Operating System" - ROS, un sistema operativo de libre distribución que corre sobre Linux, instalado en la CPU descripta como sistema de procesamiento de alto nivel. El sistema operativo ROS es ampliamente utilizado en la comunidad de diseñadores y fabricantes de robots debido a sus características de software abierto, que permite encapsular nodos desarrolla-dos en diferentes lenguajes, facilitando enormemente el crecimiento incremental del sistema y su prototipado. En efecto, la incorporación de nuevos nodos que manejen nuevos dispositivos, tanto sensores como actuadores, se realiza de forma inmediata y homogénea, aún para dispositivos con drivers realizados en software propietario y muy diferentes entre sí. En etapa de prototipado las aproximaciones se desarrollaron en Matlab( y luego de probados como estables y verificados experimentalmente, fueron pasados a $\mathrm{C}++$. Estas características le confieren a toda la arquitectura una gran versatilidad, fundamental-mente para el trabajo de investigación tecnológica.

En la Figura 3 se grafica un esquema descriptivo de la arquitectura de software del ASV MACÁBOT.

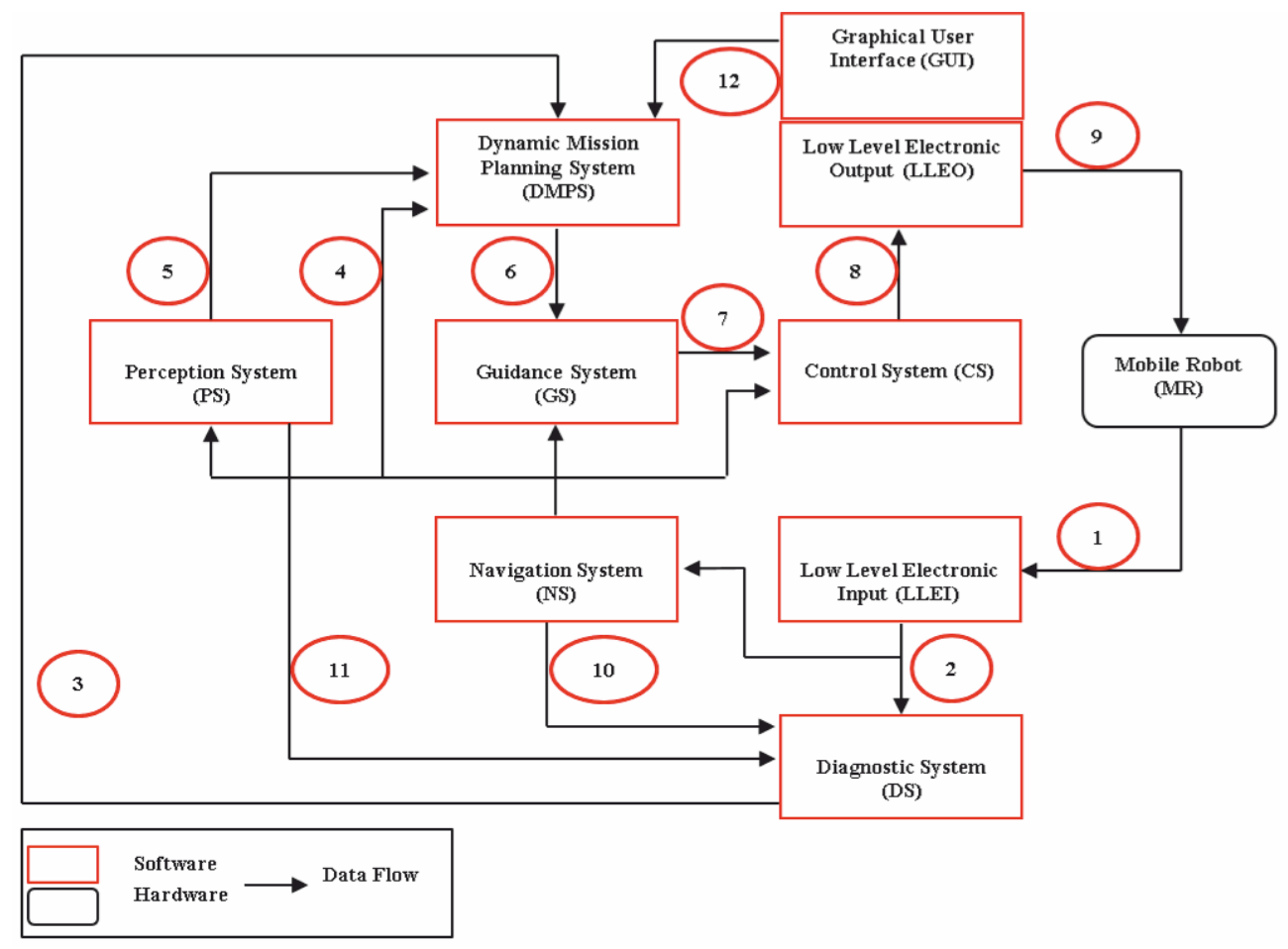

Fig. 3: Arquitectura de software del prototipo de ASV Macábot. 
El flujo de datos de cada sistema de SW/HW se puede resumir en la siguiente tabla :

\begin{tabular}{|c|c|c|}
\hline Sistema Software & Entrada & Salida \\
\hline Low Level Electronic Input (LLEI) & Autonomous Mobile Robot (1) & DS - NS (2) \\
\hline Diagnostic System (DS) & LLEI (2) - NS (10) - PS (11) & DMPS (3) \\
\hline Navigation System (NS) & LLEI (2) & CS - GS - DMPS - PS (10) \\
\hline Perception System (PS) & NS (10) & DMPS (5) \\
\hline $\begin{array}{c}\text { Dynamic Mission Planning } \\
\text { System (DMPS) }\end{array}$ & DS (3) - NS (10) - PS (5) - GUI (12) \\
\hline $\begin{array}{c}\text { Guidance System (GS) } \\
\text { Control System (CS) }\end{array}$ & NS (10) - DMPS (6) & CS (7) - NS (10) \\
\hline $\begin{array}{c}\text { Low Level Electronic Output } \\
\text { (LLEO) }\end{array}$ & CS (8) & $\begin{array}{c}\text { Autonomous Mobile Robot } \\
(9)\end{array}$ \\
\hline
\end{tabular}

Tabla 1

\section{A. Módulos LLEI y LLEO}

Son los encargados de vincular los dos niveles de electrónica con un protocolo de comunicación NMEA 0183, usual en la instrumentación marítima [8]. El módulo LLEI recibe lecturas de sensores y los transforma en los mensajes de ROS denominados tópicos, del mismo modo que el módulo LLEO traduce las variables generadas en los nodos ROS que son necesarias para el manejo de actuadores o información útil en el bajo nivel.

B. Sistema de Diagnóstico - DS

Este módulo aun no ha sido completamente implementado, pero se abordará con un sistema abductivo para determinar los modos de fallo, en forma similar a la realizada en [9], que es una técnica ya bien conocida.

C. Sistema de Navegación - NS

Este módulo se resolvió empleando un sistema de Navegación Inercial asistido por GPS (GPS Aided INS) [10]. En nuestro caso, con el uso de una brújula. El INS toma medidas de una Unidad de Medición Inercial (IMU), aceleraciones, velocidades angulares y actitud, y resuelve un sistema de ecuaciones de navegación para obtener estimaciones de posición (Latitud, Longitud y elevación) y velocidades en las direcciones Norte, Este y hacia abajo (North, East y Down o sistema NED) [11]. Debido a las inferiores especificaciones de error de las IMU de grado regular, como la empleada en el ASV MACÁBOT, respecto a las de grado táctico o de navegación, la solución obtenida por el INS acumula error rápidamente. Por esto, se emplean mediciones de sensores de asistencia y técnicas de fusión de sensores para corregir periódicamente las estimaciones del INS y mantener acotado el error de los datos de navegación obtenidos. En el presente caso se emplearon los datos de posición y velocidad de un receptor GPS y de rumbo dado por una brújula electrónica, como medidas de asistencia y un Filtro de Kalman Extendido (EKF) como algoritmo de fusión de sensores. De este modo, el NS provee datos de navegación a una frecuencia de $50 \mathrm{~Hz}$ y con resolución en posición en el orden de los centímetros. El NS fue implementado en ROS como un nodo que se subscribe a los tópicos donde se publican los datos de la IMU, el GPS y la brújula, y publica en un tópico de salida los datos de navegación. Así, cualquier otro nodo del sistema ROS que requiera dichos datos, se subscribe a este tópico para recibirlos. En la figura 4 se muestra un 
esquema de la arquitectura de nodos y tópicos ROS del NS del ASV MACÁBOT actualmente implementada.

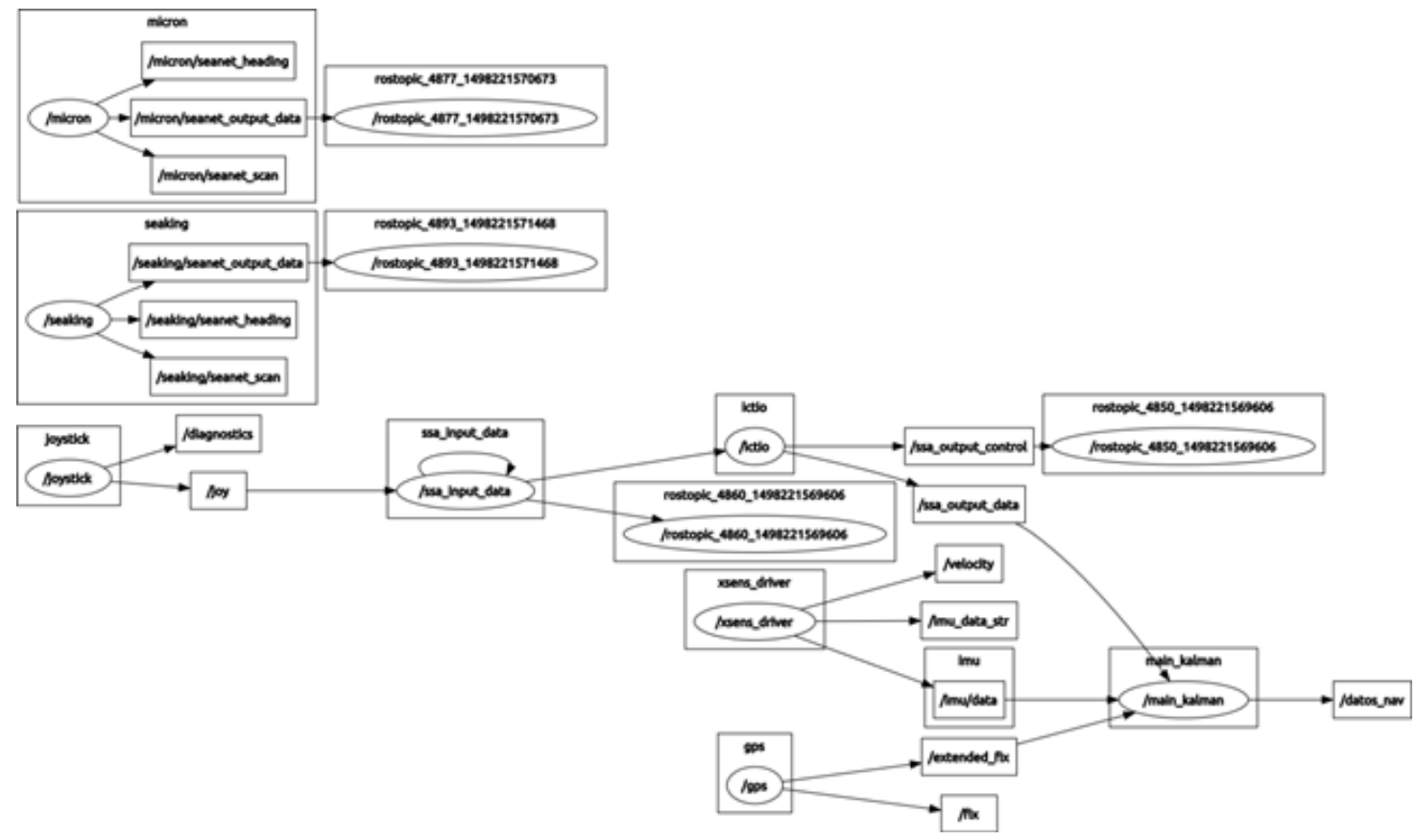

Fig. 4: Arquitectura ROS del ASV Macábot.

D. Sistema de Percepción - PS

Para tener un correcto modelo del mundo circundante, y de este modo poder tomar decisiones en forma autónoma, además de conocer con precisión acotada su propia posición y actitud, provista como se vio por el NS, el robot ha de contar con sensores que le permitan conocer la ubicación de objetos externos, que dividimos en objetos a seguir y objetos a evitar. Esta primera clasificación se realiza en este módulo mediante dos subsistemas, uno de seguimiento (Tracking Subsystem - TSS) y otro de detección de obstáculos (Obstacle Detection Subsystem - ODSS [12]). El TSS maneja los datos provenientes de ecosondas en el caso de realización de batimetrías, o de cualquier otro sensor de acuerdo a la aplicación, y debe ser capaz de realizar detecciones en tiempo eficiente [13-14]. El ODSS actual-mente emplea los datos provenientes de la ecosonda mecánicamente escaneada (Micron Tritech@ de la Fig. 2) y en un futuro próximo se combinará con un LIDAR para detección de obstáculos de superficie.

E. Sistema Planificador Dinámico de Misión - DMPS

Las trayectorias del ASV se generan como un conjunto de 4 puntos de paso o waypoints, de modo tal que el robot siempre se encuentra navegando entre el primero y el segundo. $\mathrm{Ni}$ bien el segundo es alcanzado, una nueva trayectoria se genera por este módulo, convirtiendo al segundo punto de paso en primero y así sucesivamente hasta llegar a destino. Los otros dos puntos restantes de la trayectoria se reservan como back-up frente a incidencias y trayectorias de evasión. El usuario, al programar la misión mediante el módulo GUI, provee 
de los puntos de paso principales por los que ha de pasar el robot. Sin embargo, durante el transcurso de la misma pueden surgir contingen-cias como obstáculos, merma de energía, pérdida del objetivo a inspeccionar, desarmado de la formación en caso de navegación en escuadra de varios robots, que ameritan una replanificación de las trayectorias y/o tareas predefinidas. El módulo DMPS es el responsable de esto, y lo lleva a cabo empleando una técnica también muy establecida de inteligencia artificial como lo son los sistemas basados en conocimiento [15-16].

F. Sistema de Guiado - GS

El elemento clave para la operación eficiente del sistema de control es la generación en tiempo real de sus referencias apropiadas. Esta justamente es la función de este módulo: a partir de los 4 puntos de paso provistos por el DMPS, genera una trayectoria para proveer las referencias a los controladores del CS. Para ello se emplea un algoritmo basado en Lyapunov que optimiza tiempo y velocidad de recorrido de las trayectorias, adaptado de [17]. El algoritmo considera la fuerza de atracción entre dos partículas ficticias, una dentro del robot y la otra sobre la trayectoria deseada. De la interacción de ambas se determina a) la velocidad y orientación del ASV para aproximarse a la partícula en movimiento en la trayectoria y b) el refresco de la posición de la partícula dentro de la trayectoria deseada. Su implementación se describe en detalle en [18].

G. Sistema de Control - CS

El sistema de control recibe, como se ve en la Fig. 3, dos vectores de entrada: el (10) proveniente del NS es el vector espacio de estados $\mathrm{X}$ de posiciones, velocidades y actitudes, medidas y fusionadas, y por otro lado, el (7) proveniente del GS que es el vector de estados deseados o referencias $\mathrm{X}^{*}$. De este modo, es posible cerrar lazos de realimentación negativa. En esta primera aproximación y para las pruebas que se muestran en la sección siguiente, estos lazos de control se cerraron empleando controladores de tipo PI, de un modo similar a lo realizado previamente para otros robots autónomos similares. Las ganancias de estos lazos fueron ajustadas para obtener una respuesta transitoria óptima [19].

\section{PRUEBAS EXPERIMENTALES}

Durante el pasado mes de abril de 2017 se realizaron las pruebas finales de integración de todos los módulos descriptos en secciones previas para el ASV MACÁBOT. Éstas fueron desarrolladas en un espejo de agua tranquila, formado por el embalse que el arroyo Tapalqué encuentra frente al club Estudiantes, en la ciudad de Olavarría. En las figuras 5 y 6 se aprecia la trayectoria descripta por el ASV, a partir de los datos de posición colectados desde el NS. Se puede ver en la figura 5 la corrección del módulo NS luego de fusionar los datos crudos de GPS (en azul) con los provenientes del INS y la brújula, a través del EKF.

La figura 6 por su parte, muestra esta trayectoria final superpuesta con los archivos de Google Maps@ de la zona de realización de los experimentos.

Si bien se trata de pruebas preliminares de integración en las que se validó el correcto funcionamiento de cada módulo en software y hardware, la plataforma multipropósito desarrollada demostró ser lo suficientemente robusta como para abordar posteriores estudios y aplicaciones específicas. 


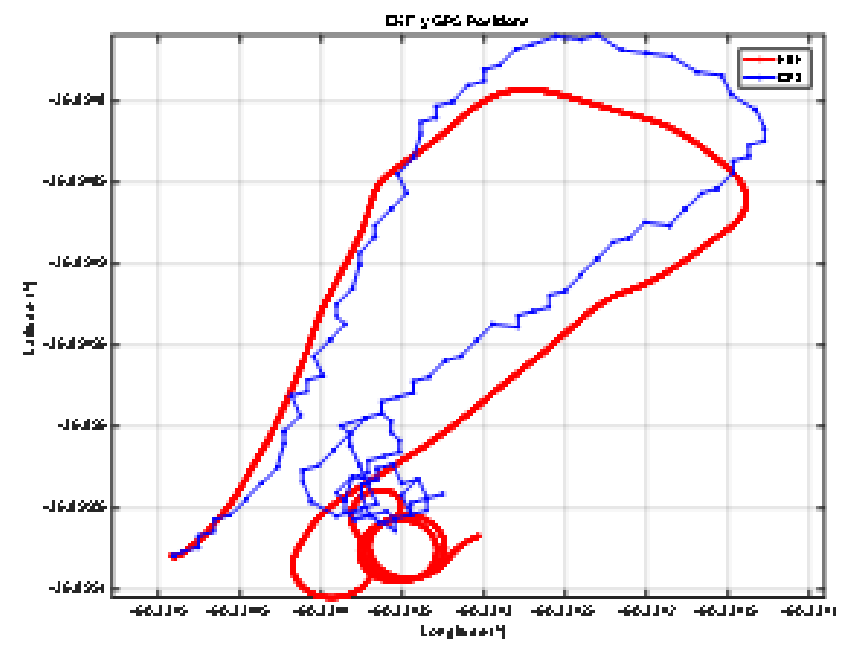

Figura 5: Medidas del GPS (azul) y datos del NS (rojo).

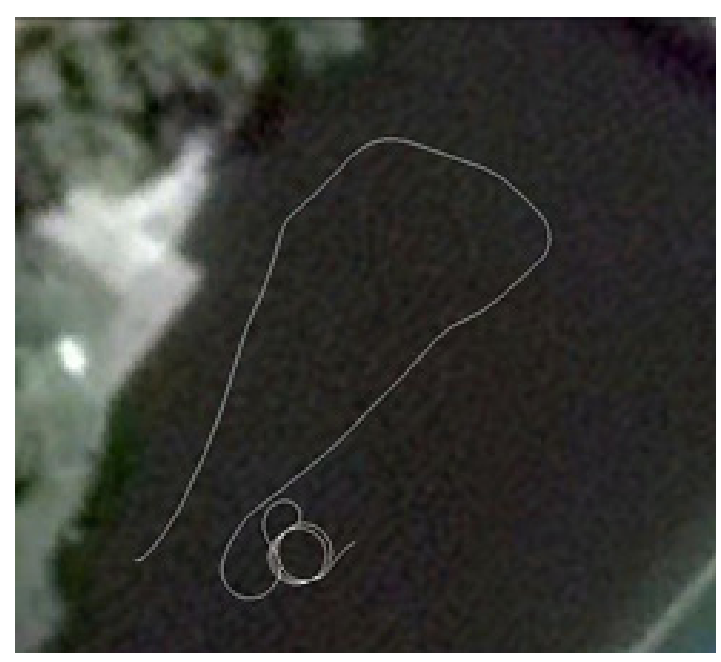

Figura 6: Recorrido de prueba del ASV MACÁBOT en el Arroyo Tapalqué (lat. -36.893996, long. -60.333412).

\section{CONCLUSIONES Y TRABAJO FUTURO}

En este trabajo se ha presentado el detalle de diseño y construcción de un vehículo autónomo de superficie (ASV) de propósito múltiple, así como también las pruebas de validación de los diferentes módulos funcionando en el agua. Estas pruebas demostraron la robustez del robot, desem-peñándose en un entorno real no estructurado. A partir de este aporte, se 
pretende asegurar por un lado la posibilidad de profundizar en el estudio y optimización de las aproximaciones realizadas para los sistemas de guiado y control, de fusión de sensores, de sistema de percepción, y de autonomía de decisiones con el sistema planificador dinámico, por ejemplo, agregando interesantes capacidades de aprendizaje y adaptación. El MACÁBOT constituye así una plataforma fácilmente portable y versátil para sustentar los experimentos necesarios para diferentes investigaciones. Por otra parte, se constató que la tecnología tal y como está, se encuentra lo suficientemente madura como para abordar diferentes aplicaciones de interés, como realizar exploracio-nes batimétricas de lechos sumergidos en forma automatiza-da, para mantenimiento de puertos o estudios hidrológicos, estudios de ecosistemas costeros, boyas virtuales para posicionamiento en redes de sensores sumergidos, entre otras. 


\section{REFERENCIAS}

[1] Pastore and Đapić, Improving Autonomy and Control of Autonomous Surface Vehicles in Port Protection and Mine Countermeasure Scenarios, Journal of Field Robotics 27(6), pp. 903-914, Wiley Periodicals, Inc. DOI: 10.1002/rob.20353, 2010.

[2] Martins, Alfredo, Almeida, J. M., Ferreira, H., Silva, H., Dias, N., Dias, A., Almeida, C., Silva, E. P., Autonomous Surface Vehicle Docking Manoeuvre with Visual Information, IEEE International Conference on Robotics and Automation, Roma, Italy, 10-14 April 2007.

[3] Caiti, Andrea, Garulli, A., Livide, F., and Prattichizzo, D., Autonomous Surface Vehicle Docking Manoeuvre with Visual Information, IEEE International Conference on Robotics and Automation, Roma, Italy, 10-14 April 2007.

[4] Acosta, Gerardo G., Curti, H., Calvo Ibáñez, O., and Rossi, S., Some Issues on the Design of a Low-Cost Autonomous Underwater Vehicle with an Intelligent Dynamic Mission Planner for Pipeline and Cable Tracking, Chapter I in Underwater Vehicles, pp. 1-18 I-Tech Online Books, Robotics Series, I-Tech Education and Publishing KG, Viena, Austria, Editor: A. Inzartsev, 2009, <Open Access: http://books.i-techonline.com/>, ISBN 978-953-7619-49-7, 2009.

[5] Fossen, Thor, Marine Control Systems. Trondheim, Norway, Marine Cybernetics Ed. 2002.

[6] Meystel, A., Autonomous Mobile Robots: Vehicles With Cognitive Control, World Scientific, 1991.

[7] Acosta, Gerardo G., Alonso, C., and Pulido B., Basic Tasks for Knowledge Based Supervision in Process Control, Eng. App. of Artificial Intelligence, Vol. 14, N ${ }^{\circ}$, Elsevier Science Ltd/IFAC, pp. 441-455. (ISSN 0952-1976), 2001.

[8] https://www.nmea.org/content/nmea_standards/nmea_0183_v_410.asp

[9] Alonso, Carlos, Acosta, G. G., Mira, J., and Prada, C., Knowledge Based Process Control Supervision and Diagnosis: the AEROLID Approach, Expert Systems with Applications Journal, vol 14, pp 371-383. ISSN: 0957-4174, 1998.

[10] Grewal, M., Weill, L., and Andrews, A., Global Positioning Systems, Inertial Navigation and Integration, Second Edi. John Wiley and Sons, 2007.

[11] D. H. Titterton and J. L. Weston, Strapdown Inertial Navigation Technology - 2nd Edition, 2nd ed. The Institute of Electrical Engineers, 2004.

[12] Villar, Sebastián, Solari, F., Menna, B., Acosta, G.G., Obstacle Detection System Design for an Autonomous Surface Vehicle using a Mechanical Scanning Sonar, aceptado para su publicación en RPIC, Mar del Plata, Septiembre 2017.

[13] Acosta, Gerardo G., and Villar, Sebastián, Accumulated CA-CFAR Process in Two Dimensions for on-line Object Detection from Side Scan Sonar Data, IEEE Journal of Oceanic Engineering, Vol. 40, N 3, pp. 558-69, ISSN 0364-9059, 2015.

[14] Villar, Sebastián, Solari, F., De Paula, M., Acosta, G.G., A Framework for Acoustic Segmentation Using OS-CFAR 2-D from Sidescan Sonar Data, IEEE Journal of Oceanic 
Engineering, Print ISSN 0364-9059, Online ISSN: 1558-1691, DOI: 10.1109/JOE.2017.2721058 (available on-line).

[15] Acosta, Gerardo G., H. J. Curti, O. Calvo Ibáñez, and S. R. Rossi, "A knowledge-based approach for AUV path planner development”, WSEAS TRANS. SYST., VOL. 5, NO. 6, PP. 1417-1424, JUN. 2006.

[16] Acosta, Gerardo G., H. J. Curti, and O. Calvo, "Autonomous underwater pipeline inspection in AUTOTRACKER PROJECT : the navigation module," presented at the IEEEOCEANS'05 Europe Conference, Brest, France, 2005, vol. 1, pp. 389-394.

[17] Breivik, M., and Fossen, T., "Principles of guidance-based path following" in 2d and 3d. 44th IEEE Conf. Decision and Control, 627-634, Sevilla, Spain, December 2005.

[18] Rozenfeld, Alejandro, Acosta, Gerardo G.G., Sousa, A., Curti, H., and O. Calvo Ibáñez, "A guidance and control system proposal for autonomous pipeline inspections," Transactions on Systems, Signals \& Devices, Vol. 5, N . 1, pp. 5-27, Shaker-Verlag, Germany, ISSN: 1861-5252, November 2010.

[19] Valenciaga, Fernando, Puleston, P., Calvo Ibáñez, O., and Acosta, G.G., "Trajectory Tracking of the 'Cormoran' AUV Based on a PI-MIMO Approach" in Proc. del IEEE/ Oceans'07 Europe Conference, Aberdeen, Scotland, June 18-21, pp. 1-6 (ISBN: 978-1-42440635-7), 2007. 